\title{
有机配体对 $\mathrm{NaBiF}_{4}: \mathrm{Yb}^{3+} / \mathbf{E r}^{3+}$ 上转换纳米粒子形貌 和发光性能的调控研究
}

\author{
郡 康，汪 涛，闻莹婷，滕渊洁，刘会君，潘再法 \\ (浙江工业大学 化学工程学院, 杭州 310014 )
}

摘 要: 本工作采用一锅溶剂热法分别制备了不同有机配体修饰的 $\mathrm{NaBiF}_{4}: \mathrm{Yb}^{3+} / \mathrm{Er}^{3+}$ 上转换纳米粒子(UCNPs), 并对 其形貌和发光性能进行了研究。实验表明, 有机配体的软模板和导向作用可调控 UCNPs 的粒径和形貌, 且有机配 体的缺陷钝化作用会使其发光增强。其中，以十六烷基三甲基溴化铵(CTAB)和十六烷基三甲基氯化铵(CTAC)修饰 的 UCNPs 的增强效果最为显著, 强度大约增加了 9 倍。此外, 本研究进一步考察了该 UCNPs 在不同的温度和 $\mathrm{pH}$ 条件下的发光强度的变化规律。结果表明, 在 30 90 ${ }^{\circ} \mathrm{C}$ 之间, 其发光强度随着温度的升高而降低; 在强酸和强碱环 境中, 其发光强度显著降低, 而在 $\mathrm{pH}$ 为 5 6 时, 其发光强度最大。

关 键 词: 上转换纳米粒子; $\mathrm{NaBiF}_{4}: \mathrm{Yb}^{3+} / \mathrm{Er}^{3+}$; 有机配体

中图分类号: O482 文献标识码: A

\section{Modulation of Morphology and Luminescence Property of $\mathrm{NaBiF}_{4}: \mathrm{Yb}^{3+} / \mathrm{Er}^{3+}$ Upconversion Nanoparticles by Organic Ligands}

\author{
SHAO Kang, WANG Tao, WEN Yingting, TENG Yuanjie, LIU Huijun, PAN Zaifa \\ (College of Chemical Engineering, Zhejiang University of Technology, Hangzhou 310014, China)
}

\begin{abstract}
NaBiF}_{4}: \mathrm{Yb}^{3+} / \mathrm{Er}^{3+}$ upconversion materials modified by different organic ligands were synthesized by solvent thermal method, and then their morphology and luminescence properties were studied. The results show that the soft template and orientation of the organic ligands can tune the particle size and morphology of the UCNPs, and the defect passivation of the surface organic ligands enhances the luminescence intensity. Especially, the cetyl trimethylammonium bromide (CTAB) and the cetyl trimethylammonium chloride (CTAC) modified materials have the most significant enhancement effect ( 9 times). Furthermore, the effects of temperature and $\mathrm{pH}$ on luminescent properties of the materials were investigated. Results show that, within the range of experimental conditions, the luminescence intensity of the materials decreases with the increase of temperature from 30 to $90{ }^{\circ} \mathrm{C}$ and significantly reduces under strong acid and alkaline conditions, with maximum at $\mathrm{pH}$ 5-6.
\end{abstract}

Key words: upconversion nanoparticles; $\mathrm{NaBiF}_{4}: \mathrm{Yb}^{3+} / \mathrm{Er}^{3+}$; organic ligand

上转换纳米粒子(UCNPs) 具有自荧光背景低、 反斯托克斯位移大、光稳定性好、穿透深度高、
毒性低、抗闪胨和光漂白能力强等许多独特的性 质 ${ }^{[1-5]}$, 因而在生物传感、生物成像、光动力治疗及

收稿日期: 2019-05-20; 收到修改稿日期: 2019-07-05

基金项目: 国家自然科学基金(21804119); 浙江省自然科学基金重点项目(LZ18B050002)

National Natural Science Foundation of China (21804119); Key Project of National Natural Science Foundation of Zhejiang Provincial (LZ18B050002)

作者简介: 邵 康(1987-), 男, 助理研究员. E-mail: sk1033@zjut.edu.cn SHAO Kang(1987-), male, lecturer. E-mail: sk1033@zjut.edu.cn

通讯作者：潘再法，副教授.E-mail: panzaifa@zjut.edu.cn PAN Zaifa, male, associate professor. E-mail: panzaifa@zjut.edu.cn 
光热治疗、光导开关和信息存储等很多领域具有广 泛的应用前景。目前为止, 为了获得较强的上转换 发光, 已经开发了氧化物、硫化物、氟氧化物、氟 化物、硼酸盐、磷酸盐、钼酸盐、钨酸盐和钒酸盐 等多种上转换材料体系 ${ }^{[6-14]}$ 。其中, 氟化物因为其晶 格声子能量相对较低, 化学稳定性较高, 能有效降 低非辐射弛豫速率, 通常具有相对较高的上转换发 光效率 ${ }^{[15]}$, 其中基于 $\mathrm{NaYF}_{4}$ 体系的 UCNPs 得到了 广泛的研究。但是其昂贵的原材料和复杂的合成步 骤限制了它们的进一步应用, 因此需要探索新型 UCNPs 体系。

铋无毒无害、成本低, 张洪杰课题组 ${ }^{[16]}$ 开发了 基于 $\mathrm{NaBiF}_{4}$ 基质的 UCNPs 体系。在室温下, 通过 简单混合摚拌 $1 \mathrm{~min}$, 便可快速制备 $\mathrm{Yb}^{3+} / \mathrm{Er}^{3+}$ 共掺 杂 $\mathrm{NaBiF}_{4}$ 基 UCNPs。之后还通过简单的溶剂热法 合成 PAA 修饰的 $\mathrm{NaBiF}_{4}: \mathrm{Yb}^{3+} / \mathrm{Er}^{3+}$, 聚丙烯酸功能 化不仅提高了 $\mathrm{NaBiF}_{4}: \mathrm{Yb}^{3+} / \mathrm{Er}^{3+}$ 上转换材料的发光 强度, 而且减小了粒子的尺寸 ${ }^{[17]}$ 。这种有机配体的 改性作用大大提升了其生物应用的潜力, 但是除了 PAA 修饰的 $\mathrm{NaBiF}_{4}: \mathrm{Yb}^{3+} / \mathrm{Er}^{3+}$ 之外, 鲜有相关的报 道。基于此, 本工作采用溶剂热法合成了几种有机 配体修饰的 $\mathrm{NaBiF}_{4}: \mathrm{Yb}^{3+} / \mathrm{Er}^{3+}$ 上转换材料并对其相 关性能进行了系统研究。

\section{1 实验方法}

\section{1 样品制备}

本研究采用溶剂热法制备几种具有不同有机配 体修饰的 $\mathrm{NaBiF}_{4}: \mathrm{Yb}^{3+} / \mathrm{Er}^{3+}$ 上转换纳米粒子。其中, 有机配体分别为聚丙烯酸 $50 \%$ 水溶液 $(\mathrm{PAA}, \mathrm{MW}=$ $3000) 、$ 聚乙烯亚胺(PEI, MW $=10000)$ 、聚乙烯吡咯 烷酮(PVP, MW=40000)、十六烷基三甲基溴化铵 (CTAB)、十六烷基三甲基氯化铵(CTAC) 以及柠檬酸 (CA)。

有机配体修饰的 $\mathrm{NaBiF}_{4}: \mathrm{Yb}^{3+} / \mathrm{Er}^{3+}$ 上转换纳米 粒子的制备: 首先按一定的化学计量比称取 $\mathrm{Bi}\left(\mathrm{NO}_{3}\right)_{3} \cdot 5 \mathrm{H}_{2} \mathrm{O}(99.0 \%) 、 \mathrm{Yb}\left(\mathrm{NO}_{3}\right)_{3} \cdot 5 \mathrm{H}_{2} \mathrm{O}(99.9 \%)$ 、 $\mathrm{Er}\left(\mathrm{NO}_{3}\right)_{3} \cdot 5 \mathrm{H}_{2} \mathrm{O}(99.9 \%)$ 以及一定量的 $\mathrm{NaNO}_{3} 、 \mathrm{NH}_{4} \mathrm{~F}$ 和有机配体, 然后将称量好的 $\mathrm{Bi}\left(\mathrm{NO}_{3}\right)_{3} \cdot 5 \mathrm{H}_{2} \mathrm{O}$ 、 $\mathrm{Yb}\left(\mathrm{NO}_{3}\right)_{3} \cdot 5 \mathrm{H}_{2} \mathrm{O} 、 \mathrm{Er}\left(\mathrm{NO}_{3}\right)_{3} \cdot 5 \mathrm{H}_{2} \mathrm{O} 、 \mathrm{NaNO}_{3}$ 和有机配 体超声分散于 $10 \mathrm{~mL}$ 乙二醇中, 记为反应母液 $\mathrm{A}$; 将 称量好的 $\mathrm{NH}_{4} \mathrm{~F}$ 超声溶解于 $20 \mathrm{~mL}$ 乙二醇中, 记为 反应母液 $\mathrm{B}$ 。在水浴 $30{ }^{\circ} \mathrm{C}$ 、剧烈搅拌的条件下, 将 上述反应母液 $\mathrm{A}$ 缓慢倾入反应母液 $\mathrm{B}$ 中, 反应 $10 \mathrm{~min}$ 后, 将产物转移到聚四氟乙烯内胆的高压反 应釜中, $200{ }^{\circ} \mathrm{C}$ 反应 $8 \mathrm{~h}$, 自然冷却至室温后, 离心
(4000 r/min, $10 \mathrm{~min}$ )收集产物, 用去离子水和无水乙醇 洗涤 3 次, 并将产物分散于 $10 \mathrm{~mL}$ 去离子水中。上述 所得不同有机配体修饰的上转换材料分别用以下符号 表示: $\mathrm{NaBiF}_{4} 、 \mathrm{PAA}-\mathrm{NaBiF}_{4} 、 \mathrm{PEI}-\mathrm{NaBiF}_{4} 、 \mathrm{PVP}-\mathrm{NaBiF}_{4}$ 、 CTAB-NaBiF 4 、CTAC-NaBiF $、$ CA-NaBiF 4 。另外, 以 同样方法合成没有配体修饰的 $\mathrm{NaBiF}_{4}: \mathrm{Yb}^{3+} / \mathrm{Er}^{3+}$ 上转 换纳米粒子, 作为空白对照组。

\section{2 性能测试}

采用 $\mathrm{KBr}$ 压片法通过傅里叶红外光谱仪 (Nicolet 6700 IR, 赛默飞世尔(中国)有限公司)测定 样品的红外光谱; 在 X 射线粉末衍射仪 ( $\chi$ 'pert PRO, 荷兰 PANlytical 公司)上测定样品的 X 射线衍射谱; 使用纳米激光粒度仪(Malvern Nano-Z, 美国马尔文 公司)测定材料的粒径分布和电势; 利用苂光光谱 仪(Fluoromax-4 Spectrofluorometer, 法国 HORIBA Jobin Yvon 公司)测试材料的发射光谱, 激发波长固 定为 $980 \mathrm{~nm}$, 由红外二极管激光器 (MDL-H980(FC)-5W, 长春新产业光电技术有限公司)提供; 用扫描电镜(4700(II), 日本 Hitachi 公司)观察材料 的形貌。

\section{2 结果与讨论}

\section{1 红外光谱的测定}

首先利用傅里叶红外光谱仪表征 $\mathrm{NaBiF}_{4}$ 、 PAA-NaBiF $、$ PEI-NaBiF 4 、 PVP-NaBiF 4 、CTAB$\mathrm{NaBiF}_{4} 、 \mathrm{CTAC}-\mathrm{NaBiF}_{4} 、 \mathrm{CA}-\mathrm{NaBiF}_{4}$ 及其相应配体 的官能团的差异来初步验证有机配体修饰是否成功, 如图 1 所示。图 1(a)中, 1710 和 $1230 \mathrm{~cm}^{-1}$ 处的吸收 峰是由 PAA 中的 $\mathrm{COOH}$ 产生; 图 1(b)中, $1460 \mathrm{~cm}^{-1}$ 是 $\mathrm{CH}_{2}$ 面内弯曲振动峰, 1650 和 $1580 \mathrm{~cm}^{-1}$ 是 $\mathrm{NH}$ 弯 曲振动峰; 图 1(c)中, $1650 \mathrm{~cm}^{-1}$ 是 $\mathrm{C}=\mathrm{O}$ 伸缩振动, $1210 \mathrm{~cm}^{-1}$ 为 $\mathrm{C}-\mathrm{N}$ 伸缩振动; 图 1(d)中, 2920 和 $2850 \mathrm{~cm}^{-1}$ 是 $\mathrm{CH}$ 的伸缩振动, $1460 \mathrm{~cm}^{-1}$ 是 $\mathrm{CTAB}$ 中 氨基的特征峰; 图 1(e)中, 2920 和 $2860 \mathrm{~cm}^{-1}$ 是 $\mathrm{CH}$ 的伸缩振动, $1450 \mathrm{~cm}^{-1}$ 是 $\mathrm{CTAC}$ 中氨基的特征峰; 图 1(f)中, 1620 和 $1390 \mathrm{~cm}^{-1}$ 是 $\mathrm{COOH}$ 的特征峰。以上 红外光谱信息可以初步表明配体修饰成功。

\section{2 结构表征}

PAA-NaBiF $、$ PEI-NaBiF $、$ PVP-NaBiF $、$ CTAB-NaBiF 4 、CTAC-NaBiF 4 和 CA-NaBiF 4 六种不 同有机配体修饰的 UCNPs 的 XRD 表征结果如图 2 所示。图 2 中可以看出, 几种材料的衍射峰与六方 相 $\mathrm{NaBiF}_{4}$ 的标准卡片(JCPDS: 41-0796)吻合, 此外, PVP-NaBiF 4 样品中“*”标记处的杂峰可能是由 $\mathrm{BiF}_{3}$ 杂相造成的。根据文献[18]报道, $\mathrm{BiF}_{3}$ 杂相也是一种 

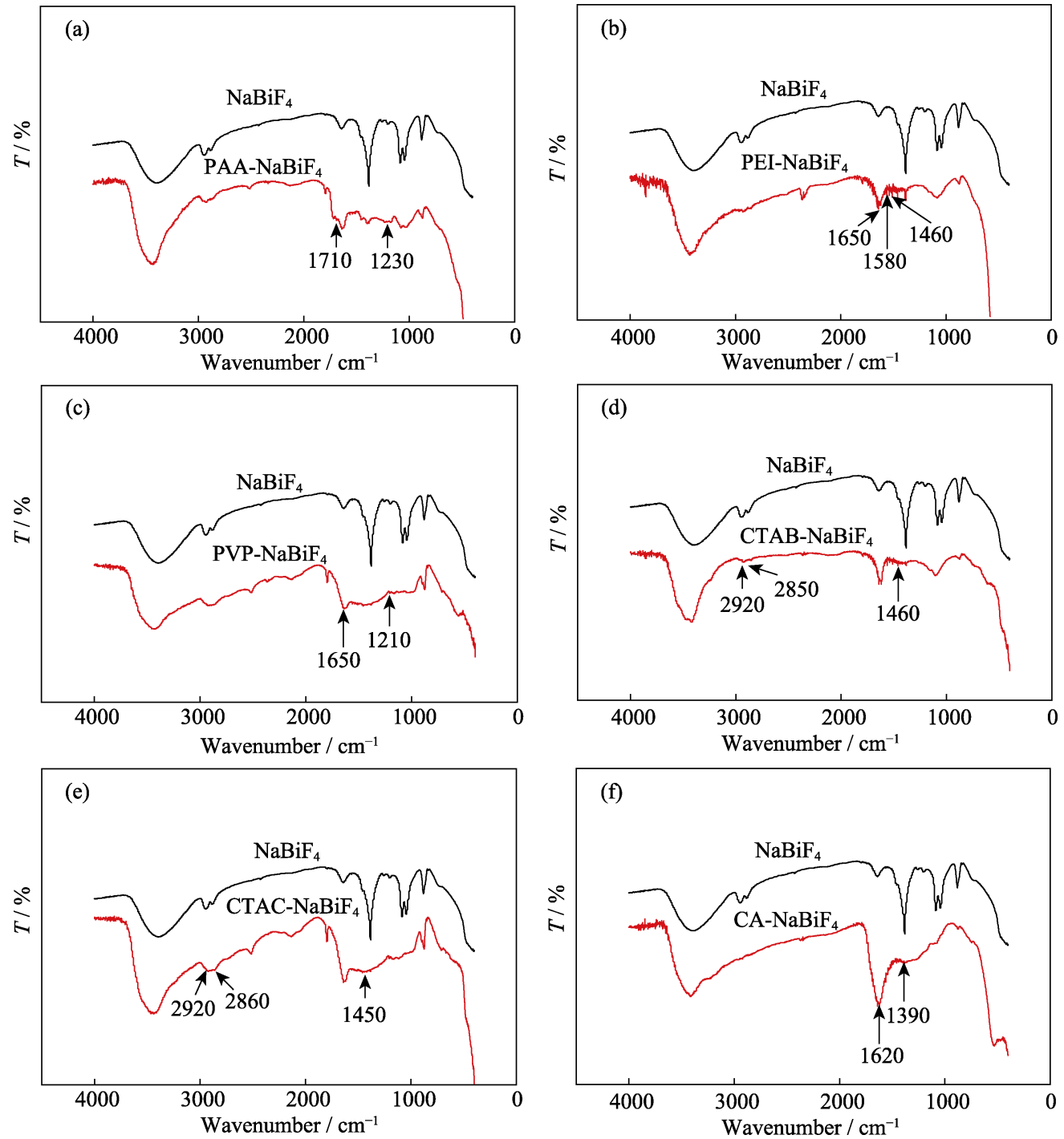

图 1 (a)PAA-NaBiF 、 (b)PEI-NaBiF $、$ 、(c)PVP-NaBiF $、$ (d)CTAB-NaBiF $、$ (e)CTAC-NaBiF 4 和(f)CA-NaBiF 4 的红外光谱图 Fig. 1 IR spectra of (a) PAA-modified $\mathrm{NaBiF}_{4}$, (b) PEI-modified $\mathrm{NaBiF}_{4}$, (c) PVP-modified $\mathrm{NaBiF}_{4}$, (d) $\mathrm{CTAB}$-modified $\mathrm{NaBiF}_{4}$, (e) $\mathrm{CTAC}$-modified $\mathrm{NaBiF}_{4}$, and (f) $\mathrm{CA}$-modified $\mathrm{NaBiF}_{4}$

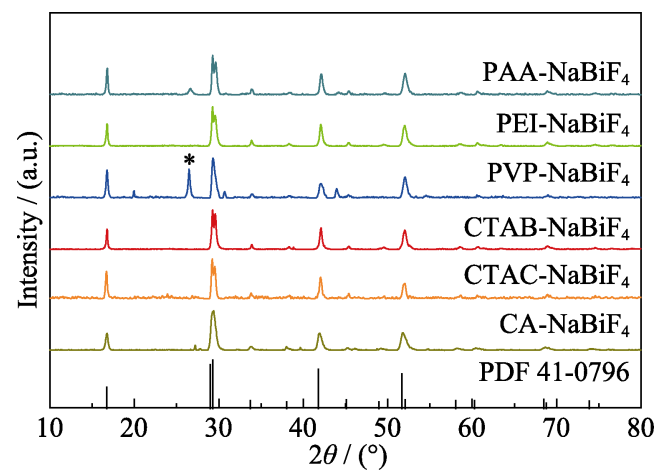

图 2 几种样品的 XRD 谱图

Fig. 2 XRD patterns of several samples

上转换材料的基质, $\mathrm{BiF}_{3}: \mathrm{Yb}^{3+} / \mathrm{Er}^{3+}$ 在 $523 \mathrm{~nm}$ 、 $545 \mathrm{~nm} 、 655 \mathrm{~nm}$ 处也有和 $\mathrm{NaBiF}_{4}: \mathrm{Yb}^{3+} / \mathrm{Er}^{3+}$ 类似的 发射峰, 只是强度相对较弱。基于此, 可以认为 $\mathrm{BiF}_{3}$ 对材料的上转换发光影响可以忽略不计。
PAA-NaBiF 4 、 $\mathrm{PEI}-\mathrm{NaBiF}_{4} 、 \mathrm{PVP}_{-} \mathrm{NaBiF}_{4}$ 、 CTAB-NaBiF $4 、 C T A C-\mathrm{NaBiF}_{4}$ 和 $\mathrm{CA}-\mathrm{NaBiF}_{4}$ 六种不 同有机配体修饰的 $\mathrm{NaBiF}_{4}: \mathrm{Yb}^{3+} / \mathrm{Er}^{3+}$ 上转换材料的 扫描电镜(SEM)照片如图 3 所示。图像中可以看出, 合成的几种 UCNPs 的形貌和尺寸各有不同, 说明有 机配体对材料的形貌具有调控作用，PAA- $\mathrm{NaBiF}_{4}$ 、 PEI-NaBiF 4 和 PVP-NaBiF 4 三种材料的尺寸相对较 小，而 CTAB-NaBiF $、$ CTAC-NaBiF 4 和 CA-NaBiF 4 三种材料的尺寸较大。初步推测有机配体的软模板 和导向作用显著影响了粒子的成核或生长过程, 导 致合成的材料的形貌出现明显差异。

用激光粒度仪对所合成的几种 $\mathrm{NaBiF}_{4}$ 基 UCNPs 的粒径进行测定, 如图 4 所示。图 4 中可以看出,

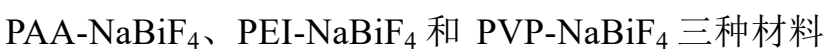
粒径相对较小, 而 CTAB-NaBiF $4 、$ CTAC- $\mathrm{NaBiF}_{4}$ 和 

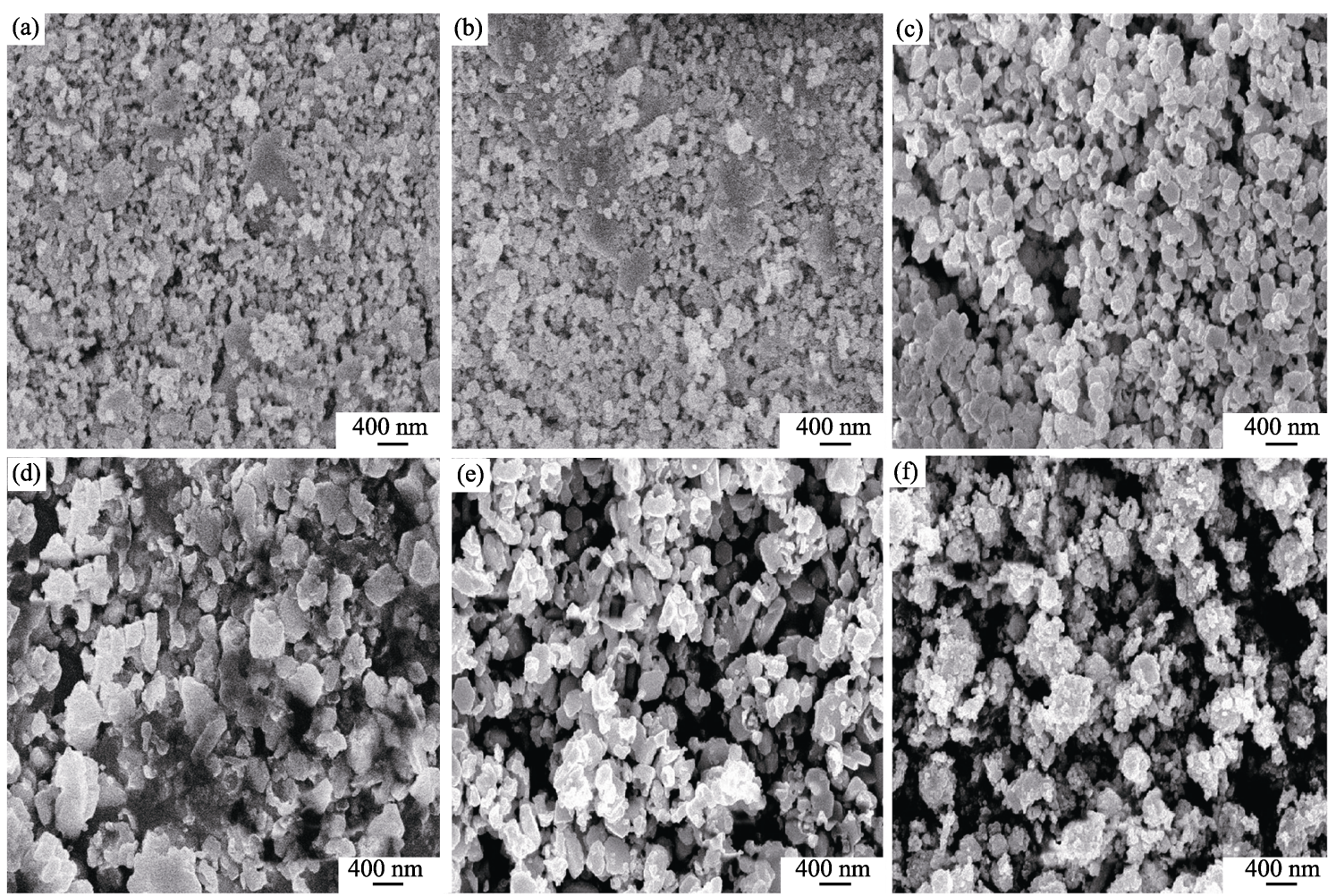

图 3 (a)PAA-NaBiF 、 (b)PEI-NaBiF 、 (c)PVP-NaBiF $、$, (d)CTAB-NaBiF $、$, (e)CTAC- $\mathrm{NaBiF}_{4}$ 和(f)CA-NaBiF 4 的扫描电镜照片 Fig. 3 SEM images of (a) PAA-modified $\mathrm{NaBiF}_{4}$, (b) PEI-modified $\mathrm{NaBiF}_{4}$, (c) PVP-modified $\mathrm{NaBiF}_{4}$,

(d) CTAB-modified $\mathrm{NaBiF}_{4}$, (e) $\mathrm{CTAC}_{\text {-modified }} \mathrm{NaBiF}_{4}$, and (f) CA-modified $\mathrm{NaBi}$
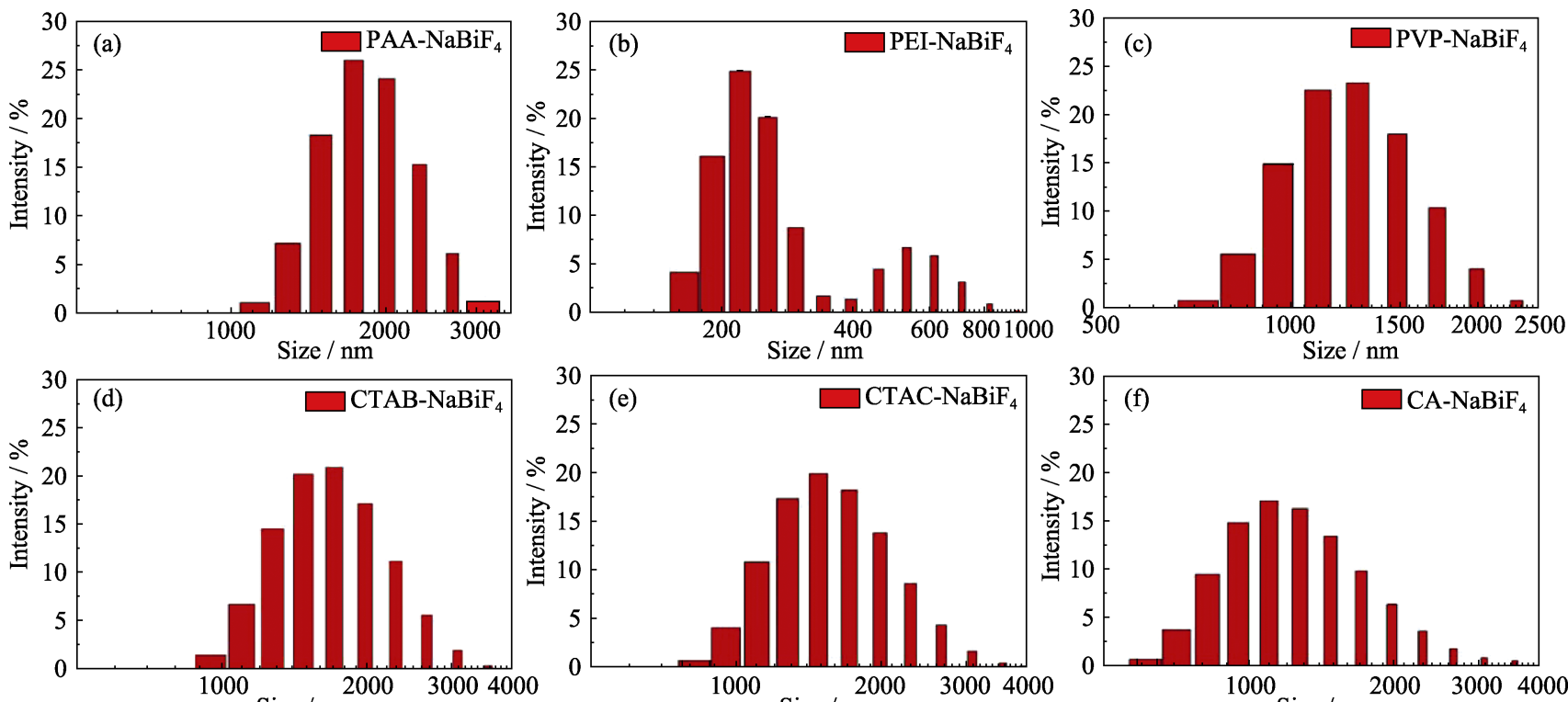

图 4 (a)PAA-NaBiF 、 (b)PEI-NaBiF 、 (c) $\mathrm{PVP}_{4} \mathrm{NaBiF}_{4}$ 、(d)CTAB-NaBiF $、$ (e)CTAC-NaBiF 4 和(f)CA-NaBiF 4 的粒径分布图

Fig. 4 Size distributions of (a) $\mathrm{PAA}-\mathrm{NaBiF}_{4}$, (b) $\mathrm{PEI}-\mathrm{NaBiF}_{4}$, (c) $\mathrm{PVP}-\mathrm{NaBiF}_{4}$, (d) $\mathrm{CTAB}-\mathrm{NaBiF}_{4}$,

(e) $\mathrm{CTAC}-\mathrm{NaBiF}_{4}$, and (f) $\mathrm{CA}-\mathrm{NaBiF}_{4}$

CA-NaBiF 4 三种材料的粒径相对较大, 结合上述 SEM 照片可以发现，前面三种材料在合成过程中大 部分的小颗粒发生了聚集，粒径分布在较小的区域; 而后面三种材料在合成过程中少部分的小颗粒发生 了聚集, 大部分则是生长为较大的颗粒, 粒径总体
分布在较大的区域。因为粒径仪测得的是水合粒径, 所以尺寸比 SEM 照片大。根据有机配体的结构、官 能团、SEM 照片以及水合粒径数据可以大致推断不 同配体对 $\mathrm{NaBiF}_{4}$ 基 UCNPs 的形貌调控机制：在金 属离子前体、有机配体和氟离子前体的混合体系中, 
有机配体能和金属离子前体发生配位结合，进而影 响氟化物晶体的成核和生长。在反应过程中有机配 体一金属离子配合物会大量形成，氟离子将通过竞 争取代这些结合的有机配体, 进而形成 $\mathrm{NaBiF}_{4}$ 晶 体。通过这些有机配体的竞争、软模板和导向作用, 实现晶体的结构和形貌调控, 进而调控其发光性 能。此外，有机配体结合在晶体表面起到钝化表面、 减少表面缺陷的作用，同样可以调控其发光性能。

六种配体的结构式如图 5 所示。其中, 含有羧 基的有机配体对 $\mathrm{Bi}^{3+} 、 \mathrm{Yb}^{3+}$ 和 $\mathrm{Er}^{3+}$ 前体有很强的结 合能力, 导致氟离子与离子前体形成晶体时的速度 变慢, 所以 $\mathrm{PAA}-\mathrm{NaBiF}_{4}$ 和 $\mathrm{CA}-\mathrm{NaBiF}_{4}$ 的粒径较小。 但由于 $\mathrm{CA}$ 的羧基数目比 $\mathrm{PAA}$ 少, 且 $\mathrm{CA}$ 在水热条 件下易于发生分子间脱水碳化, 这使得 $\mathrm{CA}$ 的配体 作用减弱，进而导致形成的 $\mathrm{CA}-\mathrm{NaBiF}_{4}$ 的尺寸略微 增大; PEI、CTAB 和 CTAC 配体中氨基与金属离子 前体的结合能力相对较弱, 氟离子易于将其竞争开, 使得氟化物晶体成核和生长较快, 形成的粒子尺 寸较大。CTAB-NaBiF 4 和 $\mathrm{CTAC}-\mathrm{NaBiF}_{4}$ 的粒子尺 寸最大。但反常的是, PEI-NaBiF 4 的水合粒径却很 小, 这是因为它在水溶液中不稳定, 容易发生解体, 导致大颗粒变成小颗粒。这种反常的水响应解体现 象与我们最近的报道结果一致 ${ }^{[19]}$; 而烷酮的结合 能力介于羧基和氨基之间, 所形成的粒子尺寸相 对适中。

\section{3 材料发光性能测定}

图 6 是功率为 $0.20 \mathrm{~W} / \mathrm{cm}^{2}$ 的 $980 \mathrm{~nm}$ 激光器激 发下利用荧光光谱仪测定的不同有机配体修饰的 $\mathrm{NaBiF}_{4}: \mathrm{Yb}^{3+} / \mathrm{Er}^{3+}$ 的发射光谱, 它的发射峰主要位于 $521 、 540$ 以及 $654 \mathrm{~nm}$ 左右，分别对应 $\mathrm{Er}$ 离子的 ${ }^{2} \mathrm{H}_{11 / 2} \rightarrow{ }^{4} \mathrm{I}_{15 / 2}$ 、 ${ }^{4} \mathrm{~S}_{3 / 2} \rightarrow{ }^{4} \mathrm{I}_{15 / 2}$ 以及 ${ }^{4} \mathrm{~F}_{9 / 2} \rightarrow{ }^{4} \mathrm{I}_{15 / 2}$ 三种能量 跃迁。同时, 有机配体的修饰对材料的发光性能产 生了十分显著的影响, PAA、PVP、CTAB 和 CTAC

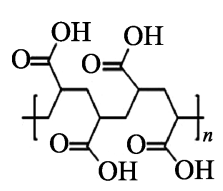

(PAA)
(PEI)

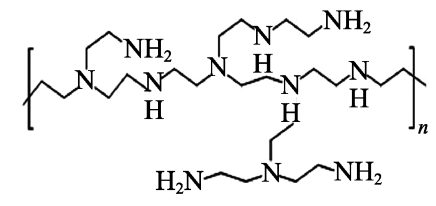

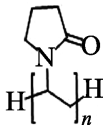

(PVP)

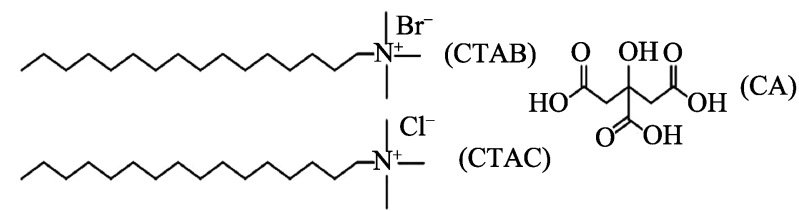

图 5 PAA、PEI、PVP、CTAB、CTAC 和 CA 六种有机配 体的结构式

Fig. 5 Structural formulas of six organic ligands: PAA, PEI, PVP, CTAB, CTAC, and CA
的成功修饰使得材料的发光强度得到了不同程度的 增强, 其中, $\mathrm{PAA}-\mathrm{NaBiF}_{4}$ 和 $\mathrm{PVP}-\mathrm{NaBiF}_{4}$ 约增强了 2 倍，而 CTAB-NaBiF $、$ CTAC- $\mathrm{NaBiF}_{4}$ 增强十分明显, 约增强了 9 倍。结合 SEM 照片、粒径分布图以及苂 光光谱图, 可以推测影响该材料上转换发光强度的 因素主要有粒子尺寸和有机配体钝化效果。随着粒 子尺寸的增大，其上转换发光强度也会增大。有机 配体针化作用减少了 UCNPs 的表面缺陷，有效防 止能量损失，从而使材料的上转换发光增强。对于 PAA-NaBiF 4 和 $\mathrm{PVP}-\mathrm{NaBiF}_{4}$ 两种材料, 粒子尺寸较 小对发光强度的负面影响小于有机配体对发光强度 的积极影响, 最终材料发光强度略微增大; 对于 CTAB-NaBiF $、$ CTAC- $\mathrm{NaBiF}_{4}$ 两种材料, 粒子尺寸 较大加上有机配体修饰两个方面的协同效应，使得 材料的发光强度有更大程度地增强。

图 6 中没有观察到 $\mathrm{PEI}-\mathrm{NaBiF}_{4}$ 和 $\mathrm{CA}-\mathrm{NaBiF}_{4}$ 材料明显的发射峰: 其中, $\mathrm{CA}-\mathrm{NaBiF}_{4}$ 呈黑色，这可 能是因为在 $200{ }^{\circ} \mathrm{C}$ 反应釜中反应时，柠檬酸被碳化 所致，且由于材料本身呈黑色，会吸收其他颜色的 光, 所以不能测得其发射光谱; 而 PEI-NaBiF 4 的发 射光谱极弱, 是因为水的淬灭作用导致。将合成的 PEI 修饰的材料溶解于无水乙醇中，在 $980 \mathrm{~nm}$ 激光 激发下，用荧光光谱仪测得如图 7 所示的发射光谱, 证明了水作为溶剂会使 PEI-NaBiF 4 发生苂光猝灭, 这与本课题组最近报道的研究结果一致 ${ }^{[19]}$ 。

\section{4 温度对材料发光性能的影响}

温度往往会对材料发光性能有较大影响, 因此需要对材料的温度依赖性进行考察。为使结 果易于观察, 选择相同条件下发光强度最大的 CTAB- $\mathrm{NaBiF}_{4}$ 和 CTAC- $\mathrm{NaBiF}_{4}$ 作为研究对象, 结果 如图 8 所示。在 30 90 ${ }^{\circ} \mathrm{C}$ 范围内, CTAB- $\mathrm{NaBiF}_{4}$ 和 $\mathrm{CTAC}-\mathrm{NaBiF}_{4}$ 的发光强度随着温度的升高均呈下降

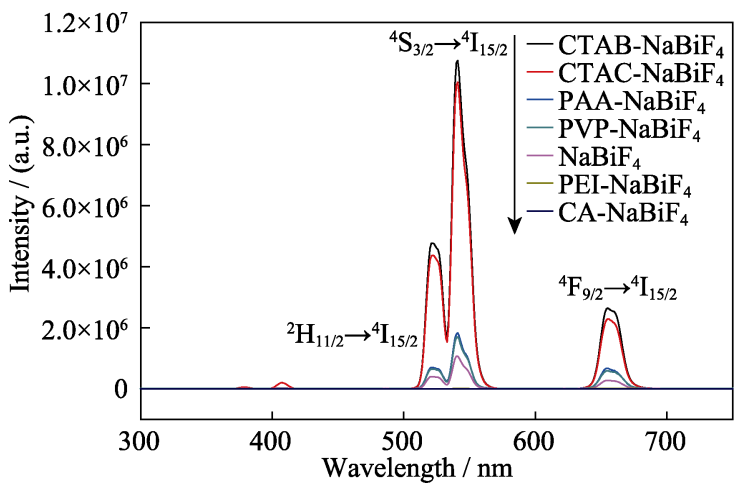

图 6 激发波长为 $980 \mathrm{~nm}$ 的不同有机配体修饰的 $\mathrm{NaBiF}_{4}$ 上 转换材料的发射光谱

Fig. 6 Emission spectra of $\mathrm{NaBiF}_{4}$ upconversion material modified by different organic ligands excited at $980 \mathrm{~nm}$ 


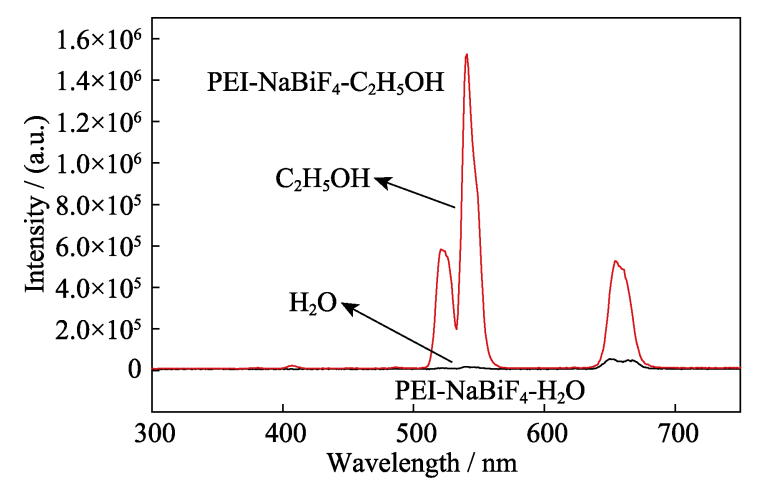

图 7 PEI-NaBiF 4 上转换材料在水和乙醇中的发射光谱

Fig. 7 Emission spectra of PEI-NaBiF 4 upconverting material in water and ethanol, respectively

趋势，温度的升高会导致能级之间的交叉弛豫过程 和无辐射跃迁几率增大 ${ }^{[17]}$, 与此同时, 温度的升高
也会使上转换纳米粒子发生一定的分解，形成小颗 粒, 从而使上转换发光强度降低。对 $540 \mathrm{~nm}$ 处的发 射峰 $\left({ }^{4} S_{3 / 2} \rightarrow{ }^{4} I_{15 / 2}\right)$ 强度与温度进行作图, 可以更加 明显地观察到上述结果。

\section{$2.5 \mathrm{pH}$ 对材料发光性能的影响}

$\mathrm{pH}$ 是另一个可能影响材料发光性能的重要因 素。本文选择 PAA-NaBiF $、$ PVP-NaBiF $、$ CTAB-NaBiF 4 和 $\mathrm{CTAC}-\mathrm{NaBiF}_{4}$ 四种材料进行 $\mathrm{pH}$ 依赖性研究, 结 果如图 9 所示。从图中可以看出, $\mathrm{pH}$ 为 3 11 范围内, 几种材料的发光强度均呈现先增强后减弱的变化趋 势, 在 $\mathrm{pH}$ 为 5 6 时, 发射强度均达到最大值。根据 本课题组之前的报道 ${ }^{[19]}$, 在强酸或者强碱环境下, $\mathrm{NaBiF}_{4}: \mathrm{Yb}^{3+} / \mathrm{Er}^{3+}$ 材料会发生一定的分解，导致粒子 不完整, 从而使上转换发光强度大幅度降低。对
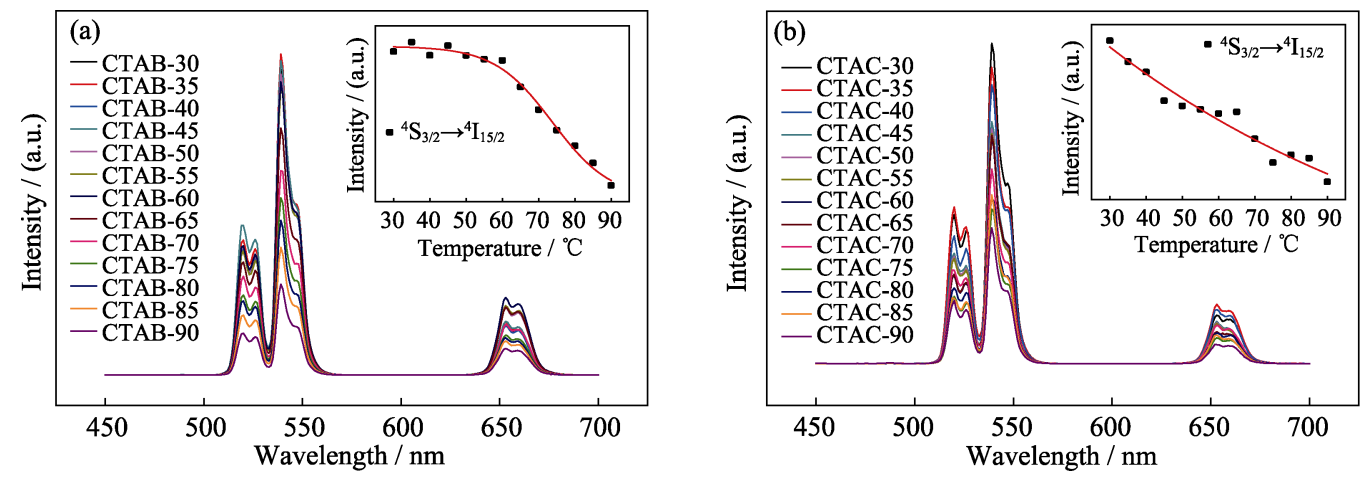

图 8 (a)CTAB- $\mathrm{NaBiF}_{4}$ 和(b)CTAC- $\mathrm{NaBiF}_{4}$ 材料在不同温度下的发射光谱

Fig. 8 Emission spectra of (a) $\mathrm{CTAB}-\mathrm{NaBiF}_{4}$ and (b) $\mathrm{CTAC}-\mathrm{NaBiF}_{4}$ at different temperatures
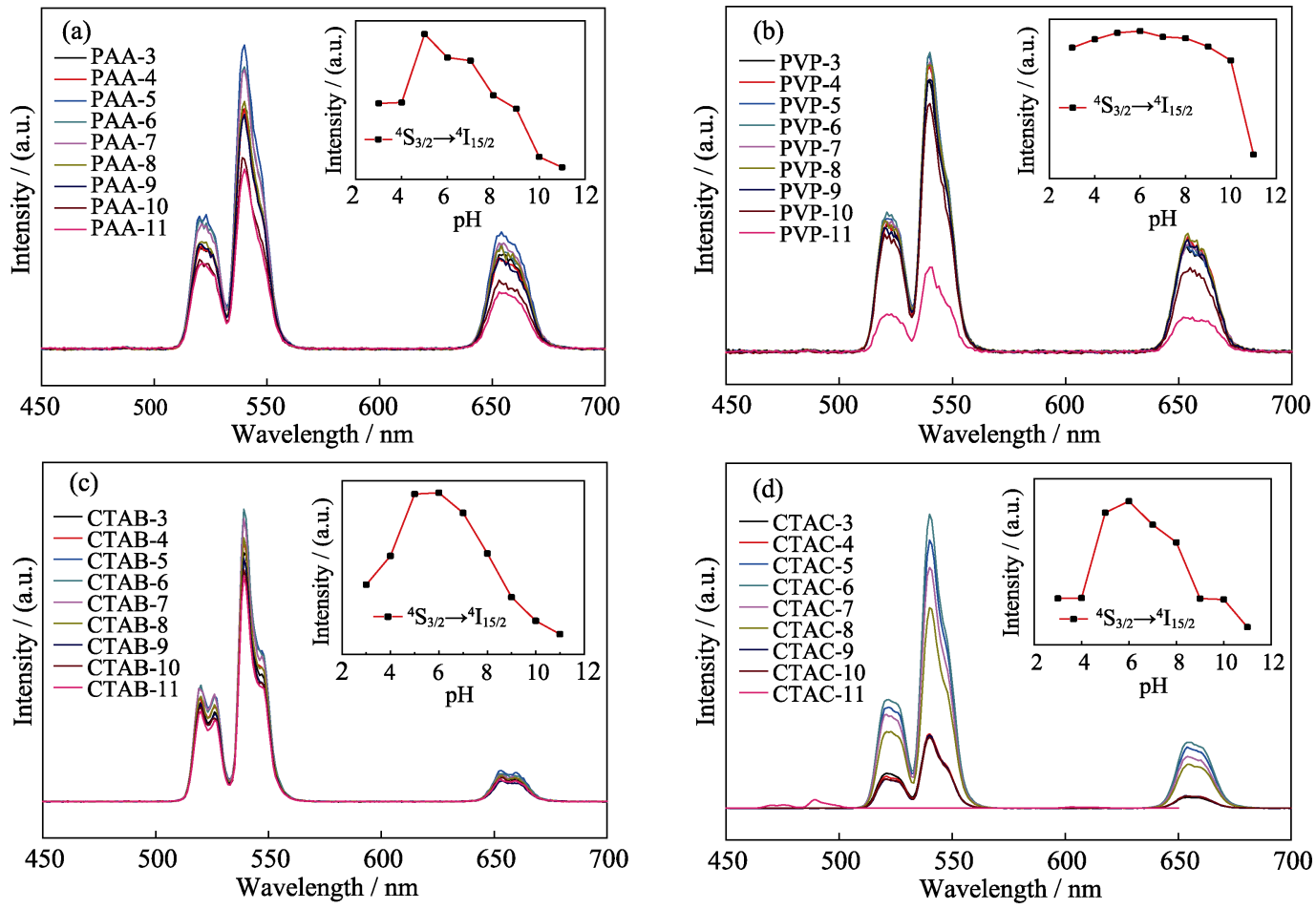

图 9 (a)PAA- $\mathrm{NaBiF}_{4}$ 、(b) $\mathrm{PVP}-\mathrm{NaBiF}_{4} 、(\mathrm{c}) \mathrm{CTAB}-\mathrm{NaBiF}_{4}$ 和(d)CTAC-NaBiF 4 材料在不同 $\mathrm{pH}$ 条件下的发射光谱 Fig. 9 Emission spectra of (a) $\mathrm{PAA}-\mathrm{NaBiF}_{4}$, (b) $\mathrm{PVP}-\mathrm{NaBiF}_{4}$, (c) $\mathrm{CTAB}-\mathrm{NaBiF}_{4}$, and (d) $\mathrm{CTAC}_{-} \mathrm{NaBiF}_{4}$ under different $\mathrm{pH}$ conditions 
$540 \mathrm{~nm}$ 处的发射峰 $\left({ }^{4} \mathrm{~S}_{3 / 2} \rightarrow{ }^{4} \mathrm{I}_{15 / 2}\right)$ 强度与 $\mathrm{pH}$ 值进行 作图(图 9 插图), 可以更加明显地观察到上述结果。 对于 $\mathrm{PAA}-\mathrm{NaBiF}_{4} 、 \mathrm{CTAB}-\mathrm{NaBiF}_{4}$ 和 $\mathrm{CTAC}-\mathrm{NaBiF}_{4}$ 三种材料, 其发光强度的上升和下降趋势都比较明

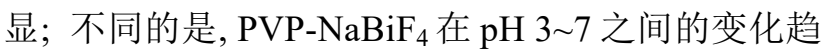
势较不明显，但其在碱性环境下则明显呈下降趋势， 在 $\mathrm{pH}$ 为 11 时下降十分显著。总体来看, 四种材料 在强酸性和强碱性条件下的发光强度都会有较明显 的下降，而且碱性环境的影响大于酸性环境。

\section{3 结论}

本研究通过简单的溶剂热法合成了 $\mathrm{PAA}-\mathrm{NaBiF}_{4}$ 、 PEI-NaBiF $、$ PVP-NaBiF $、$ CTAB-NaBiF $、$ CTAC-NaBiF 4 和 $\mathrm{CA}-\mathrm{NaBiF}_{4}$ 六种有机配体修饰的 $\mathrm{NaBiF}_{4}$ 基 UCNPs。 实验结果表明用有机配体修饰的 $\mathrm{NaBiF}_{4}$ 上转换材 料与未修饰的材料相比, 其粒径和形貌可通过不同 的配体修饰进行调控，且发光性能得到明显增强。 同时, 本研究也讨论了配体对 $\mathrm{NaBiF}_{4}$ 材料的改性机 制, 为得到性能更好的 $\mathrm{NaBiF}_{4}$ 基上转换材料提供了 新的思路。此外, 材料的苂光强度受温度和 $\mathrm{pH}$ 的影 响较大, 通过优化实验, 证明在较低温度和偏中性 条件中荧光强度最大, 为材料的进一步应用提供了 参考。

\section{参考文献:}

[1] HUANG P, ZHENG W, ZHOU S Y, et al. Lanthanide-doped $\mathrm{LiLuF}_{4}$ upconversion nanoprobes for the detection of disease biomarkers. Angewandte Chemie International Edition, 2014, 53(5): 1252-1257.

[2] GORRIS H H, WOLFBEIS O S. Photon-upconverting nanoparticles for optical encoding and multiplexing of cells, biomolecules, and microspheres. Angewandte Chemie International Edition, 2013, 52(13): 3584-3600.

[3] ZHOU J, LIU Q, FENG W, et al. Upconversion luminescent materials: advances and applications. Chemical Reviews, 2015, 115(1): 395-465.

[4] IDRIS N M, GNANASAMMANDHAN M K, ZHANG J, et al. In vivo photodynamic therapy using upconversion nanoparticles as remote-controlled nanotransducers. Nature Medicine, 2012, 18(10): $1580-1585$.

[5] TIAN G, GU Z Z, ZHOU L J, et al. $\mathrm{Mn}^{2+}$ dopant-controlled synthesis of $\mathrm{NaYF}_{4}: \mathrm{Yb} / \mathrm{Er}$ upconversion nanoparticles for in vivo imaging and drug delivery. Advanced Materials, 2012, 24(9): $1226-1231$.

[6] LEI P P, LIU X L, DONG L L, et al. Lanthanide doped $\mathrm{Bi}_{2} \mathrm{O}_{3}$ upconversion luminescence nanospheres for temperature sensing and optical imaging. Dalton Trans., 2016, 45(6): 2686-2693.

[7] LUO X X, CAO W H. Upconversion luminescence of holmium and ytterbium co-doped yttrium oxysulfide phosphor. Materials Letters, 2007, 61(17): 3696-3700.

[8] DU Y P, ZHANG Y W, SUN L D, et al. Luminescent monodisperse nanocrystals of lanthanide oxyfluorides synthesized from trifluoroacetate precursors in high-boiling solvents. Journal of Physical Chemistry C, 2008, 112(2): 405-415.

[9] BOYER J C, CUCCIA L A, CAPOBIANCO J A. Synthesis of colloidal upconverting $\mathrm{NaYF}_{4}: \mathrm{Er}^{3+} / \mathrm{Yb}^{3+}$ and $\mathrm{Tm}^{3+} / \mathrm{Yb}^{3+}$ monodisperse nanocrystals. Nano Letters, 2007, 7(3): 847-852.

[10] DAS EDDY A A, PRAKASH G V. Strong green upconversion emission from $\mathrm{Er}^{3+}-\mathrm{Yb}^{3+}$ codoped $\mathrm{KCaBO}_{3}$ phosphor. Chemical Physics Letters, 2011, 504(4/5/6): 206-210.

[11] HEER S, LEHMANN O, HAASE M, et al. Blue, green, and red upconversion emission from lanthanide-doped $\mathrm{LuPO}_{4}$ and $\mathrm{YbPO}_{4}$ nanocrystals in a transparent colloidal solution. Angewandte Chemie, 2003, 42(27): 3179-3182.

[12] DU H Y, LAN Y J, XIA Z G, et al. Synthesis and upconversion luminescence properties of $\mathrm{Yb}^{3+} / \mathrm{Er}^{3+}$ codoped $\mathrm{BaGd}_{2}\left(\mathrm{MoO}_{4}\right)_{4}$ powder. Materials Research Bulletin, 2009, 44(8): 1660-1662.

[13] XU W, ZHAO H, LI Y X, et al. Optical temperature sensing through the upconversion luminescence from $\mathrm{Ho}^{3+} / \mathrm{Yb}^{3+}$ codoped CaWO 4. Sensors \& Actuators B Chemical, 2013, 188(11): 1096-1100.

[14] SUN Y J, LIU H J, WANG X, et al. Optical spectroscopy and visible upconversion studies of $\mathrm{YVO}_{4}: \mathrm{Er}^{3+}$ nanocrystals synthesized by a hydrothermal process. Chemistry of Materials, 2006, 18(11): 2726-2732.

[15] CHEN G Y, OHULCHANSKYY T Y, KACHYNSKI A, et al. Intense visible and near-infrared upconversion photoluminescence in colloidal $\mathrm{LiYF}_{4}: \mathrm{Er}^{3+}$ nanocrystals under excitation at $1490 \mathrm{~nm}$. ACS Nano, 2011, 5(6): 4981-4986.

[16] LEI P P, AN R, YAO S, et al. Ultrafast synthesis of novel hexagonal phase $\mathrm{NaBiF}_{4}$ upconversion nanoparticles at room temperature. Advanced Materials, 2017, 29(22): 1700505.

[17] LEI P P, AN R, ZHAI X S, et al. Benefits of surfactant effects on quantum efficiency enhancement and temperature sensing behavior of $\mathrm{NaBiF}_{4}$ upconversion nanoparticles. Journal of Colloid and Interface Science, 2017, 5(37): 9659-9665.

[18] SARKAE S, DASH A, MAHALINGAM V. Strong stokes and upconversion luminescence from ultrasmall $\mathrm{Ln}^{3+}$-doped $\mathrm{BiF}_{3}(\mathrm{Ln}=$ $\left.\mathrm{Eu}^{3+}, \mathrm{Yb}^{3+} / \mathrm{Er}^{3+}\right)$ nanoparticles confined in a polymer matrix. Chemistry-An Asian Journal, 2014, 9(2): 447-451.

[19] PAN Z F, WEN Y T, WANG T, et al. One-step synthesis of hollow PEI-NaBiF $4: \mathrm{Yb}^{3+} / \mathrm{Er}^{3+}$ upconversion nanoparticles for waterresponsive luminescent probe. Journal of Rare Earths, DOI: 10.1016/j.jre.2019.04.022. 